\title{
Sete elementos do processo de seleção de casos: contribuições para um maior rigor e transparência nas Ciências Sociais
}

Daniel Baldin Machado 1,2 (1)

DOI: 10.1590/0103-3352.2021.36.243087

\section{Introdução}

Há pouco mais de duas décadas, uma parcela significativa da literatura sobre metodologia nas ciências sociais - e, talvez em maior escala, na ciência política - passou a direcionar sua atenção para os possíveis pontos fracos que as pesquisas da área poderiam possuir em suas lógicas inferenciais. Nesse período, que alguns chegam a nomear como uma era de "revolução metodológica" (MORAVCSIK, 2014, p. 48), um elemento em particular passou a ser considerado como fundamental: a necessidade de um maior rigor cientifico, principalmente nos estudos de caráter mais qualitativo (LUPIA; ELMAN, 2014; MORSE, 2018; TRACY, 2010).

No Brasil, essa discussão se deu em grande medida em torno da ideia do "calcanhar metodológico" apresentada por Gláucio Soares (2005). De maneira semelhante ao debate ocorrido internacionalmente, o autor também conclamava a necessidade de uma maior qualidade metodológica nas pesquisas conduzidas por cientistas do país, as quais deveriam deixar de possuir um

1 Mestre em Ciência Política pela Universidade Federal de São Carlos (UFSCar), Pós-Graduação em Ciência Política, São Carlos, SP, Brasil. E-mail: baldinmachado@gmail.com

2 Editor Adjunto da Revista Agenda Política, São Carlos, SP, Brasil. 
caráter mormente ensaístico para adotar o rigor como norte a ser alcançado, independentemente de a pesquisa ser quantitativa, qualitativa ou de métodos mistos. No entanto, o desenlace desse debate no Brasil tomou alguns rumos particulares, seja ao reforçar a necessidade de que essa demanda por mais rigor pudesse ser atendida com uma maior adoção de métodos quantitativos (NEIVA, 2015), seja ao sugerir que a superação dessa debilidade metodológica poderia se dar com uma modificação no currículo dos cursos de pós-graduação em ciência política no país (BARBERIA; GODOY; BARBOZA, 2014).

O presente artigo, por sua vez, insere-se nos debates de âmbito local e internacional ao buscar contribuir para a construção de pesquisas nas ciências sociais com um maior grau de rigor, mas com uma abordagem mais pragmática: visa apresentar aos pesquisadores que considerem adequado utilizar um método em particular - os estudos de caso - os principais componentes relacionados à seleção de casos, os quais poderiam servir de base para a reflexão sobre o emprego deste método em suas investigações científicas, caso busquem obter um grau mais elevado de solidez e transparência, de modo a minimizar possíveis críticas com relação a eventuais vieses em seu processo inferencial.

Para tal finalidade, o artigo encontra-se organizado em duas principais partes. A primeira delas aborda a importância da mobilização de estudos de caso - aqui entendidos tanto como análises de casos únicos ou como uma comparação entre um pequeno número de casos (BENNETT, 2004, p. 21) para as pesquisas em ciências sociais e, principalmente, para a construção de um relativo consenso sobre a necessidade de um maior rigor quando da utilização desse método.

Na sequência, são apresentados sete elementos que poderiam compor um processo rigoroso de seleção de casos: avaliação da adequação do uso de estudos de caso à pergunta de pesquisa; avaliação do tipo de objetivo de pesquisa; consideração sobre a pergunta "este caso é um caso de quê"; reflexão sobre as causas do fenômeno de estudo; consolidação da população de casos; escolha proposital de casos; e o compromisso em garantir a transparência durante todo o processo de seleção de casos. Isso é construído a partir de uma revisão bibliográfica narrativa de diversas obras da literatura que se propõem a detalhar como estudos de caso poderiam ser empreendidos, atrelando essa proposição ao debate mais amplo quanto à necessidade de se conferir 
um maior rigor às pesquisas sociais. Por fim, nas considerações finais são retomados os principais pontos apresentados, assim como são evidenciadas as principais contribuições do artigo.

\section{Por um maior rigor no processo de seleção de casos}

Ainda que seja possível dizer que a utilização de estudos de caso seja tão antiga quanto a própria existência de registros históricos, o seu emprego enquanto método científico de consolidação do conhecimento nas ciências sociais pode ser atribuído a um período bem mais recente (GEORGE; BENNETT, 2005 , p. 5). Um dos principais marcos dessa nova etapa, que alterou significativamente a maneira como os estudos de caso passaram a ser percebidos, foi a publicação de Designing Social Inquiry, de King, Keohane e Verba (1994). Essa obra iniciou um intenso debate sobre a possibilidade de pesquisas qualitativas também contribuírem com a compreensão de lógicas causais em fenômenos sociais - algo que vinha sendo relegado exclusivamente a pesquisas de caráter quantitativo -, de tal maneira que, a partir de então, todo um corpo teórico passou a ser construído em resposta a ela, expandindo significativamente a literatura que trata de metodologia em ciências sociais, o que inclui diversas discussões relativas aos estudos de caso (BEACH; PEDERSEN, 2016, p. 1-2).

Nesse processo, verificou-se que esse método teria diversos pontos fortes em sua utilização, podendo ser mobilizado justamente para contrabalancear eventuais deficiências de pesquisas demasiadamente formais. Como exemplo, George e Bennett (2005, p. 19-22) argumentam que seriam quatro as principais vantagens dos estudos de caso: a possibilidade que oferece de aumentar a validade causal de uma pesquisa, ao facilitar o reconhecimento e a mensuração de representações dos conceitos teóricos que orientam os pesquisadores; seu potencial de identificação de hipóteses a partir de sua visão aprofundada de uma instância; sua capacidade de explorar a operação pormenorizada de mecanismos causais previamente identificados; e, por fim, sua aptidão para lidar com relações de causalidade complexa, como a equifinalidade e a dependência da trajetória.

De maneira complementar, Maoz (2002, p. 162-164) avalia outras dimensões das possíveis vantagens do emprego de estudos de caso, as quais ajudariam a explicar os motivos pelos quais esse método seria tão utilizado em 
pesquisas relacionadas à política. Dentre essas vantagens, o autor sugere, por exemplo, que os estudos de caso seriam mais adequados para pesquisas que buscam compreender contextos sociais, ao possibilitar a explicitação de um processo de forma a construir uma história; que eles seriam particularmente úteis para revelar não eventos e não comportamentos e suas respectivas características; e que eles poderiam contribuir para a comparação sistemática, ao permitir a combinação, dentro de um mesmo desenho de pesquisa, de análises exploratórias e confirmatórias.

No entanto, considerando todas essas vantagens apresentadas, é de se indagar: por qual razão alguns autores ainda teriam motivos para afirmar que os estudos de caso teriam sido considerados por muito tempo como os "parentes pobres", dentre todos os métodos disponíveis nas ciências sociais (YIN, 2001, p. XI)? Ou ainda, por quais razões alguns mal-entendidos sobre os estudos de caso - como alguns muito graves, como a suposta impossibilidade de contribuir com o desenvolvimento científico - teriam subsistido por um período significativo de tempo na percepção de membros de determinadas disciplinas, em especial das ciências sociais (FLYVBJERG, 2006, p. 220)?

A resposta a essa indagação, de acordo com alguns autores, não estaria necessariamente na compreensão das possibilidades do método em si, mas sim na análise do uso que teria sido feito dele no decorrer do tempo. AlvesMazzotti (2006, p. 639), por exemplo, sugere que essa desqualificação poderia ser atribuída à utilização indevida da terminologia "estudo de caso", já que diversos pesquisadores teriam a utilizado sem uma devida caracterização ou aplicação, muitas vezes ignorando a complexidade derivada da escolha que esse método enseja, o que teria contribuído para propagar a ideia de ceticismo relacionada ao seu emprego - algo que inclusive poderia ser verificado empiricamente em diversas pesquisas brasileiras (VITORINO; OLIVEIRA, 2012). Opinião semelhante é compartilhada por Flyvbjerg (2006, p. 242), para quem a ausência de estudos de caso executados com rigor teria ajudado a disseminar exemplos negativos de como pesquisas que empregam esse método deveriam ser conduzidas nas ciências sociais, o que teria prejudicado o desenvolvimento metodológico relativo às pesquisas do campo.

Segundo Maoz (2002, p. 162), esse uso impróprio dos estudos de caso impactaria significa e negativamente na possibilidade de se tecer inferências, 
apontando pelo menos três processos relacionados ao método que poderiam ser mais bem endereçados para remediar essa questão: a seleção de casos, o desenho de pesquisa dos estudos de caso e as comparações entre casos. A primeira dessas três questões seria uma das principais responsáveis por transmitir a impressão de que, ao se optar pela utilização de estudos de caso, o autor estaria isento da necessidade de documentar quaisquer considerações metodológicas relativas à sua inferência causal, o que possibilitaria "saltos" lógicos muitas vezes derivados de uma seleção de casos enviesada (MAOZ, 2002, p. 164-166).

Esse problema, conhecido na literatura como viés de seleção, isto é, "erro sistemático resultante de inferências inadequadas formuladas a partir de uma amostra"3 (LEUFFEN, 2007, p. 146, tradução nossa), é apontado como um dos pontos nevrálgicos no que se refere ao emprego de estudos de caso, especialmente em estudos provenientes de uma tradição qualitativa (BENNETT, 2004; GEDDES, 1990; KING; KEOHANE; VERBA, 1994; SEKHON, 2004). Contudo - e afortunadamente -, também tem sido cada vez mais expressiva a quantidade de autores que passaram a considerar que esse problema não é insolúvel. Nessa linha, uma das melhores formas que vêm sendo advogadas para se lidar com essa questão é a de se comprometer com a garantia da transparência na produção acadêmica, isto é, explicar ao leitor como foram feitas as escolhas da pesquisa quanto às evidências, teorias e métodos da pesquisa (MORAVCSIK, 2014, p. 49).

A transparência com relação às escolhas feitas pelo pesquisador - que, além do detalhamento das etapas da produção acadêmica, também pode envolver os processos de coleta de dados e análise (LUPIA; ELMAN, 2014, p. 21) - não somente permite que o leitor tenha uma maior compreensão dos possíveis vieses da pesquisa, mas igualmente estimularia os pesquisadores a conduzirem, voluntariamente, pesquisas menos enviesadas (MORAVCSIK, 2014, p. 49). Isso, por sua vez, situa-se dentro de um movimento mais amplo na literatura metodológica relativo a pesquisas qualitativas que vem ocorrendo desde os anos 2000, o qual reforça a necessidade de que os pesquisadores deliberadamente tenham consciência dos limites dos resultados apresentados, contribuindo, desse modo, para a construção de um maior rigor científico dentro de cada pesquisa (MORSE, 2018, p. 1384).

3 No original, "a systematic error that results from improper inferences drawn from a sample". 
Sendo assim, a próxima seção visa oferecer sugestões práticas sobre como conduzir um processo de seleção de casos com rigor e transparência desde as primeiras etapas da pesquisa, de modo a mitigar - ou, no mínimo, evidenciar possíveis limitações inferenciais. É válido frisar que essa discussão é particularmente relevante na literatura metodológica, que considera possível escolher casos de maneira proposital, isto é, de maneira não aleatória. Por essa razão, grande parte dos autores mobilizados para os fins do presente artigo se inserem nessa vertente do debate, que acredita que é possível escolher (e não sortear) $\operatorname{casos}^{4}$.

Todavia, mesmo sem deixar de considerar as significativas contribuições dos autores alinhados à bibliografia que trata da seleção proposital (KING; KEOHANE; VERBA, 1994; LEUFFEN, 2007; LEVY, 2008; SEAWRIGHT; GERRING, 2008), suas orientações geralmente partem do pressuposto de que o pesquisador já lidou com algumas considerações anteriores ao processo de escolha dos casos, algo que é verificado ao se tentar implementar empiricamente suas técnicas. De tal sorte, torna-se necessário evidenciar todos os processos relacionados à escolha propriamente dita para que, ao final desse intenso processo de reflexão, os casos que ilustrarão as inferências causais do pesquisador possam, enfim, ser selecionados de maneira rigorosa e transparente.

\section{Sete elementos do processo de seleção de casos}

Alguns autores, como King, Keohane e Verba (1994, p. 139), argumentam que, para que seja possível selecionar casos, seria fundamental que os valores de algumas variáveis fossem compreendidos de maneira antecipada. De maneira similar, Seawright e Gerring (2008, p. 296) condicionam suas técnicas de seleção a conhecimentos prévios por parte do pesquisador - seja de suas principais inferências, seja da população de casos disponíveis para escolha. Esses exemplos indicam que, embora a utilização da expressão "seleção de casos" na literatura metodológica esteja, em geral, relacionada a uma ação específica (o ato de se escolher algumas instâncias dentre uma população de casos), existiria uma necessidade de que algumas outras atitudes fossem tomadas antes de efetivamente se proceder à escolha dos casos.

Por essa razão, optou-se, neste artigo, pelo emprego de duas diferentes nomenclaturas. $\mathrm{O}$ ato de analisar uma população de casos e, a partir dela,

$4 \quad$ Algo que será exposto de maneira pormenorizada ao se tratar do elemento que discorre sobre a escolha de casos. 
escolher um ou mais unidades para ser estudada em profundidade - como ilustrado pelos autores acima mencionados - será aqui denominado escolha de casos. Esta ação seria somente um dos componentes de um processo mais amplo, composto por diversos outros elementos além desse, que aqui receberá a denominação de seleção de casos. Portanto, ao se fazer menção ao "processo de seleção de casos", o que se pretende sugerir é que ele seria composto por pelo menos seis elementos que se apresentariam em uma ordem relativamente sequencial, acompanhado por um elemento sincrônico a todos os outros, totalizando sete elementos.

É escassa a produção literária que aborde a ideia de que a seleção de casos configura um processo mais abrangente dessa mesma maneira. $\mathrm{O}$ trabalho que mais aparenta se aproximar dessa organização e sistematização, ainda que com consideráveis diferenças, é a obra de George e Bennett (2005, p. 7388 ), embora a proposta dos autores seja outra: servir de referência à construção de desenhos de pesquisa que utilizem estudos de caso. Por essa razão, o presente artigo busca suprir essa lacuna na produção bibliográfica e, para tal, empreende uma revisão bibliográfica narrativa com a finalidade de integrar o conhecimento de uma determinada área do conhecimento (BAUMEISTER; LEARY, 1997, p. 312), a qual, neste caso, é a dos estudos sobre metodologia nas ciências sociais ${ }^{5}$.

Mais especificamente, o intuito proposto ao se referenciar diferentes autores é a organização de uma literatura preexistente, reunindo aqueles que lidam com diferentes elementos do processo de seleção de casos (como aqui entendido), e relacionando-os de maneira a possibilitar uma compreensão mais intuitiva de como selecionar casos na prática. Ademais, o presente estudo avança no sentido de sugerir um item suplementar, muitas vezes relevado durante o desenvolvimento das seções metodológicas nas investigações científicas: a necessidade de transparência. O resultado desse empreendimento, sistematizado na Figura 1, é a apresentação de alguns elementos nem sempre mobilizados

5 Quanto a esse ponto, é válido destacar que, embora muitos dos autores e autoras mobilizados no artigo possam ser relacionados à ciência política, suas produções visam contribuir com o desenvolvimento metodológico das ciências sociais como um todo. Como exemplos, a obra de King, Keohane e Verba (1994) é direcionada para "pesquisas sociais" em geral, assim como a de George e Bennett (2005) busca tratar do uso de estudos de caso nas ciências sociais em sua totalidade. De maneira similar, o argumento apresentado no presente artigo visa fomentar uma discussão sobre rigor e transparência que pretende ir além dos (nem sempre claros) limites disciplinares da ciência política, e, por esta razão, optou-se no decorrer do texto pela referência às ciências sociais de uma maneira mais abrangente. 
durante o processo de seleção de casos, os quais poderão ser referenciados por pesquisadores que decidam optar pela utilização de estudos de caso em suas pesquisas e que desejem fortalecer o rigor de suas inferências causais.

Figura 1 - Representação esquemática dos sete elementos da seleção de casos

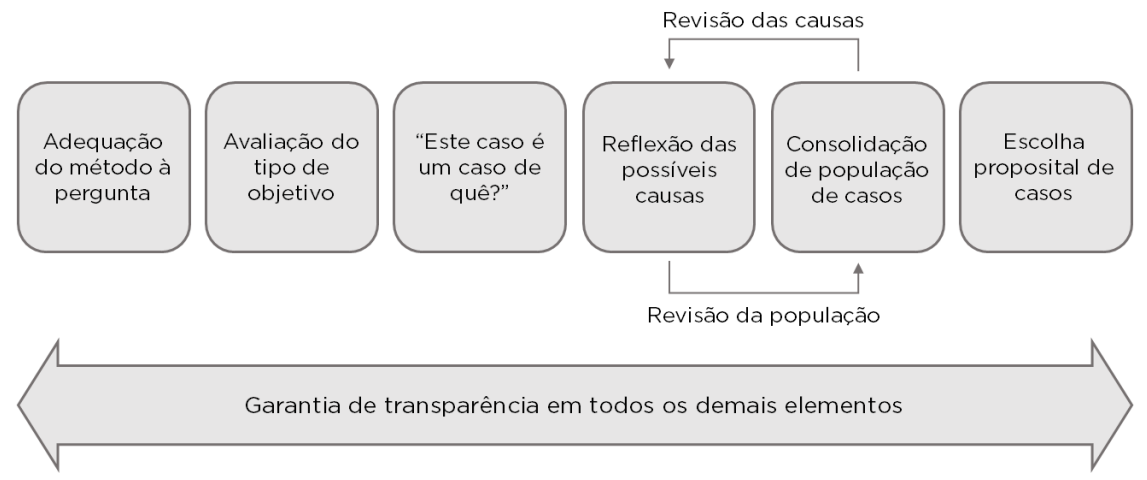

Fonte: elaboração própria.

Explicitados os objetivos e métodos da presente seção, as subseções apresentadas na sequência tratam de apresentar um maior aprofundamento sobre cada um dos sete elementos propostos, iniciando-se por um dos pontos de partida das investigações científicas: a pergunta de pesquisa.

Elemento 1: Avaliar a adequação da utilização de estudos de caso à pergunta de pesquisa

Uma das primeiras tarefas ao se começar a desenhar uma pesquisa científica nas ciências sociais é definir uma pergunta de pesquisa. King, Keohane e Verba (1994, p. 13), por exemplo, entendem que, analiticamente, o mais adequado para se estruturar o que se deseja estudar seria dividir os desenhos de pesquisa em quatro componentes, os quais deveriam ser avaliados de maneira pormenorizada: pergunta de pesquisa, teoria, dados e o uso que se faz dos dados. De modo mais detalhado, Gschwend e Schimmelfennig (2004, p. 3) argumentam que uma pesquisa empírica teria que considerar em sua elaboração algumas especificações, o que envolve definir a pergunta de pesquisa e o problema, explicitar os conceitos e a teoria, operacionalizar e medir, selecionar casos e observações, 
controlar as explicações alternativas e formular conclusões teóricas a partir da análise empírica.

Como se nota, em ambos os casos a pergunta de pesquisa é um componente fundamental e anterior à escolha do método a ser utilizado. Como argumenta Tracy (2010, p. 840), as pesquisas que visam apresentar uma maior coerência no que pretendem demonstrar deveriam possuir um alinhamento entre os objetivos perseguidos pelo pesquisador e os métodos e procedimentos utilizados para as finalidades propostas. Antes, portanto, de efetivamente considerar se estudos de caso serão utilizados para desenvolver um determinado projeto de pesquisa, faz-se fundamental avaliar se esse método é o mais adequado para a questão que é colocada, o que envolve entender pelo menos duas de suas principais aplicações de acordo com a literatura especializada.

Com esse intuito, é pertinente observar as definições do que se entende por "estudo de caso", segundo diferentes autores. Schwandt e Gates (2018, p. 602) operacionalizam uma compilação dessas definições e demonstram como o que aproxima a acepção que cada autor faz desse conceito seria o grau de profundidade da análise empreendida: os qualificadores "aprofundado", "detalhado", "complexo" e "multifacetado" são recorrentemente utilizados para se referir ao que está sendo pesquisado, isto é, "estudo", "investigação", "compreensão" e "exame”. Em outras palavras, a comparação entre conceitos ajuda a elucidar que um primeiro e possível indicador para avaliar se um pesquisador poderia ou não optar por realizar estudos de caso equivale a um autoquestionamento: a investigação visa compreender um dado fenômeno em profundidade, indo além de uma compreensão unilateral ou simplificadora daquele objeto? Caso a resposta seja afirmativa, esse seria um primeiro indicativo da aplicabilidade de um estudo de caso para compreender aquela questão.

Quem parece melhor traduzir essa ideia de busca por complexidade em termos práticos é Yin (2001, p. 26), ao sugerir que a principal maneira de avaliar se estudos de caso poderiam ser considerados uma boa escolha metodológica seria observando o tipo de questão proposta. Segundo ele, perguntas de pesquisa iniciadas com as palavras "como" ou "por que" possuiriam uma probabilidade maior de que o objetivo do pesquisador estivesse relacionado à compreensão aprofundada de alguns fenômenos no decorrer do tempo. Nesses cenários - e principalmente nas situações nas quais o pesquisador 
não poderia controlar as variáveis de seu objeto de estudo -, estudos de caso seriam uma das melhores maneiras de se entender um dado fenômeno, mobilizando múltiplas fontes de dados para tal finalidade, como, por exemplo, documentos e entrevistas (YIN, 2001, p. 27). Sendo assim, um segundo indicador da possibilidade de utilização de estudos de caso estaria no tipo de pergunta feita, e indagações iniciadas com, por exemplo, "quem", "o que”, "onde”, "quantos" e "quanto" deveriam considerar a possibilidade de utilização de outros métodos, levando em conta que outras estratégias poderiam ser mais eficientes, a depender do tipo de questão posta (YIN, 2001, p. 25).

Uma vez definida a pergunta de pesquisa e compreendido se sua resposta poderia ser perscrutada com a utilização de estudos de caso, a primeira das reflexões relacionadas ao processo de seleção de casos teria sido feita, possibilitando a passagem para a subsequente avaliação, que envolve a compreensão do tipo de objetivo de pesquisa.

\section{Elemento 2: Avaliar o tipo de objetivo de pesquisa}

Um segundo elemento relacionado ao processo de selecionar casos guarda relação com o desenho de pesquisa proposto. Uma vez conhecida a pergunta de pesquisa e verificado se a escolha metodológica por estudos de casos está adequada a ela, uma nova definição se tornaria necessária: especificar o objetivo do estudo aprofundado de um ou mais casos na pesquisa. A busca por essa resposta, por sua vez, é fundamental na medida em que os processos de escolha dos casos variam significativamente de acordo com essa resposta.

Como aponta Levy (2008, p. 3), a categorização de tipos de estudos de caso de acordo com seus objetivos não é algo recente e, inclusive, estaria disponível na literatura desde a década de 1970. Contudo, a disseminação dessas tipologias teria ocorrido sem que se contestasse uma importante característica: elas não teriam segregado os objetivos de certos tipos de estudos de caso de suas técnicas de seleção. Assim, de modo a promover essa separação, o autor sugere quatro tipos de estudos de caso de acordo com os seus objetivos: estudos de caso idiográficos, cuja finalidade seria entender um único fenômeno com maior profundidade, mas sem o intuito de propor generalizações mais amplas; estudos de caso geradores de hipóteses; estudos de caso testadores de hipóteses; e estudos de caso utilizados como sondagens de plausibilidade, cuja 
finalidade seria fazer uma investigação-piloto de hipóteses ou teorias mais amplas, antes de se despender tempo e recursos de pesquisa nessas suposições ${ }^{6}$.

Já Schwandt e Gates (2018, p. 607), empreendendo esforços para sistematizar a bibliografia relacionada ao método, encontram pontos de aproximação entre diferentes autores no que tange aos diferentes usos dos estudos de caso e, a partir disso, propõem uma tipologia composta por quatro categorias: estudos de caso descritivos, cujo objetivo seria desenvolver um retrato pormenorizado de algum fenômeno, sem necessariamente relacionar essa investigação a aspectos teóricos ou empíricos mais amplos; estudos de caso para gerar hipóteses e desenvolver teorias; estudos de caso para testar hipóteses e teorias; e, finalmente, estudos de caso que visam contribuir com teorias normativas, cujo objetivo seria analisar casos para, a partir deles, oferecer aportes relacionados a normas, valores e ideias, e não contribuições relacionadas a aspectos empíricos de um fenômeno?

Como se nota, não existe uma concordância completa com relação às possibilidades de classificação de estudos de caso. Sendo assim - e visando estabelecer um consenso mínimo entre as possibilidades apresentadas -, torna-se válido destacar também a contribuição de Yin (2001, p. 18), que classifica os estudos de caso de acordo com três distintos propósitos de pesquisa: eles poderiam ser descritivos, isto é, detalhando descritivamente os eventos de um caso no decorrer do tempo; explanatórios, visando verificar como explanações previamente estabelecidas seriam adequadas a certos eventos; e exploratórios, cujo propósito seria desenvolver hipóteses. Esses três tipos se aproximam significativamente de algumas das categorias apresentadas pelos autores mencionados anteriormente, sendo, portanto, possível determinar que existiriam pelo menos três categorias de estudos de caso de acordo com os objetivos de uma pesquisa.

A primeira categoria seria a dos estudos de caso descritivos. O termo "descritivo" provém de Schwandt e Gates (2018, p. 607) e de Yin (2001, p. 22), ainda que o conceito de estudos de caso idiográficos de Levy (2008, p. 4) também possa ser contemplado neste grupo. De maneira resumida, esse

6 No original, essas categorias seriam, respectivamente, "idiographic case studies", "hypothesis-generating case studies", "hypothesis testing case studies" e "plausibility probes" (LEVY, 2008, p. 3, tradução nossa).

7 No original, essas categorias seriam, respectivamente, "descriptive case studies", "hypothesis generation and theory development", "hypothesis and theory testing" e "contributions to normative theory" (SCHWANDT; GATES, 2018, p. 607, tradução nossa). 
tipo de estudo de caso é indicado para pesquisadores cujo desenho de pesquisa tenha por objetivo entender a complexidade de um fenômeno (ou de uma quantidade reduzida de fenômenos), sem necessariamente propor generalizações, relações causais ou construções teóricas. Em geral, sua estrutura e seu desenvolvimento possuem pouca conexão com teorias previamente estabelecidas, mas formulações teóricas podem ser utilizadas para ajudar a direcionar a atenção do pesquisador para alguns dos principais fatos do caso analisado, ainda que seu objetivo principal não seja validar empiricamente essas teorias.

Uma segunda classificação de estudos de caso de acordo com os objetivos do pesquisador seriam os estudos de caso que visam gerar hipóteses, cuja denominação provém das tipologias de Levy (2008, p. 5) e de Schwandt e Gates (2018, p. 610), e nos quais poderiam ser enquadrados os estudos de casos exploratórios segundo a classificação de Yin (2001, p. 23). Pesquisas cuja finalidade principal envolvem a elaboração de hipóteses podem perseguir esse objetivo a partir da análise aprofundada de um ou mais casos e, a partir disso, propor a generalização de seus resultados, oferecendo hipóteses passíveis de serem testadas e potencialmente validadas em fases mais avançadas de uma investigação científica ou em pesquisas posteriores. Em geral, estudos de caso são considerados muito úteis para pesquisas que têm por objetivo a geração de hipóteses, mas a sua adequação à finalidade acarreta um maior risco de possíveis vieses de seleção. Isso, por sua vez, acaba por demandar um maior rigor no processo de seleção de casos, considerando que os casos selecionados para análise poderiam estar diretamente relacionados com os objetivos da pesquisa (SCHWANDT; GATES, 2018, p. 610).

Por fim, uma terceira categoria poderia ser denominada estudos de caso que visam testar hipóteses, referenciando-se as definições apresentadas por Levy (2008, p. 6) e por Schwandt e Gates (2018, p. 612). O conceito de Yin (2001) sobre estudos de caso explanatórios também seria pertencente a esse grupo, que fundamentalmente trata das análises dentro de ou entre casos, mobilizados como método de testar e validar hipóteses. Vale destacar que o uso de estudos de caso para pesquisas com essa finalidade não é inconteste, ainda que progressivamente a literatura tenha passado a considerar que o método pode ser considerado adequado para alcançar esse objetivo, paralelamente 
ao desenvolvimento de técnicas de pesquisa rigorosas, como o mapeamento de processo e a análise qualitativa comparada ${ }^{8}$.

Ante essas possibilidades, um pesquisador, ao optar pelo uso rigoroso de estudos de caso, deveria avaliar em qual desses três grupos sua investigação poderia ser enquadrada, considerando que essa delimitação, derivada de seus objetivos de pesquisa, irá incidir sobre reflexões ensejadas por outros elementos do processo de seleção de casos, como se verá mais adiante? ${ }^{9}$.

\section{Elemento 3: Responder à pergunta "este caso é um caso de quê?"}

Um terceiro elemento da seleção de casos envolve compreender a dimensão de generalização proposta pela pesquisa. Antes, contudo, de buscar a resposta a essa questão, é preciso delimitar teoricamente o que se entende por caso e também por estudos de caso. Quanto ao primeiro ponto, é importante destacar que a tentativa de contemplar a multiplicidade de definições que diferentes pesquisadores poderiam atribuir à definição de um "caso" poderia fazer com que se chegasse à infrutífera conclusão de que uma conceitualização única seria impossível de ser alcançada (RAGIN, 1992, p. 16). Todavia, o acúmulo bibliográfico relacionado a esse método possibilitou que uma alternativa pudesse ser apontada para solucionar esse impasse: buscar compreender a finalidade para a qual os casos - e seus respectivos estudos - deveriam ser utilizados enquanto ferramenta metodológica. A partir desse ponto de partida, é possível indicar uma relativa concordância de que um caso poderia ser entendido como "uma instância de uma classe de eventos"10 (LEVY, 2008, p. 2 , tradução nossa), isto é, possuiria características que permitiriam classificá-lo em uma categoria mais abrangente de eventos ou fenômenos. Nesse sentido, um caso não seria algo isolado, de tal maneira que a principal pergunta motivadora de pesquisadores que utilizassem casos como referências seria a seguinte: "este caso é um caso de quê?"11 (LEVY, 2008, p. 2; RAGIN, 1992, p. 6; SCHWANDT; GATES. 2018, p. 601, tradução nossa).

8 No original, "process tracing" e "qualitative comparative analysis", respectivamente (SCHWANDT; GATES, 2018, p. 612-613, tradução nossa).

9 Optou-se, por uma questão de simplicidade, não incluir as categorias não consensuais na presente análise. Caso fosse necessário, no entanto, as sondagens de plausibilidade de Levy $(2008$, p. 6) poderiam ser consideradas uma forma de se testar hipóteses, assim como as contribuições à teoria normativa de Schwandt e Gates (2018, p. 615) poderiam ser inseridas dentro das possibilidades de se utilizar estudos de caso para gerar teorias.

10 No original, "an instance of a class of events".

11 No original, em cada uma das obras referenciadas, "what is this a case of?". 
Pacificado esse primeiro ponto, o segundo conceito a exigir clarificação seria o de estudo de caso. Com relação a essa questão, uma forma de sintetizar o debate existente seria dividindo a interpretação de diferentes autores em dois grupos. Dentro do primeiro deles poderia ser enquadrada, por exemplo, a definição apresentada por Borges (2007, p. 48), que interpreta que os estudos de caso seriam um método direcionado à investigação de "um fenômeno contemporâneo dentro do seu contexto, especialmente quando os limites entre o fenômeno e o contexto não são claros" (BORGES, 2007, p. 48). Essa concepção de estudos de caso enquanto um conjunto de eventos situados no tempo e no espaço, todavia, parece estar relegada a algumas disciplinas específicas, como a história, ao passo que em outras, como na ciência política, o enfoque tem sido no entendimento de fenômenos específicos como uma maneira de construir e validar proposições teóricas mais abrangentes (LEVY, 2008, p. 2).

A partir dessa premissa de generalização dos casos para categorias mais abrangentes, é possível entender o conceito de caso como uma instância de um fenômeno mais amplo, podendo-se, ainda, definir estudos de caso como

\begin{abstract}
[...] a análise intensiva (qualitativa ou quantitativa) de uma única unidade ou de um pequeno número de unidades (os casos), na qual o objetivo do pesquisador é entender uma classe mais abrangente de unidades similares (uma população de casos) ${ }^{12}$. (SEAWRIGHT; GERRING, 2008, p. 296, tradução nossa).
\end{abstract}

Em outras palavras, a ideia principal seria que o pesquisador refletisse a respeito do que busca compreender com sua pesquisa, algo que pode ser encontrado ao se buscar a resposta a uma questão fundamental: "este caso é um caso de quê?".

Considerar essa indagação como um dos elementos do processo de seleção de casos, por sua vez, resulta na necessidade de se retomar os diferentes tipos de objetivos de pesquisa apresentados anteriormente. Como se viu, nem todos os tipos de análise se propõem a construir uma relação entre os achados de um caso e explicações mais abrangentes. Os estudos de caso descritivos, por

12 No original, "the intensive (qualitative or quantitative) analysis of a single unit or a small number of units (the cases), where the researcher's goal is to understand a larger class of similar units (a population of cases)". 
exemplo, restringem-se a oferecer um detalhamento pormenorizado de um fenômeno específico sem, necessariamente, propor generalizações a partir dele e, em sendo assim, não poderiam responder à questão proposta no presente item $^{13}$. Por exclusão, os tipos de estudo de caso que estariam sujeitos à necessidade de compreensão de seu papel generalizante seriam apenas dois: os estudos de caso que visam gerar hipóteses e aqueles que visam testar hipóteses ${ }^{14}$.

Para essas duas categorias de análise, portanto, entender o que o caso representa nas discussões mais amplas é fundamental. Um exemplo ilustrativo pode ser encontrado na obra States and Social Revolutions, de Theda Skocpol, na qual a autora se propõe a compreender as causas das revoluções sociais. Amparada no estudo de três casos - as Revoluções Francesa, Russa e Chinesa -, ela esclarece que seus objetivos "não estão meramente na narração de cada um dos casos, mas principalmente no entendimento e na explicação da lógica generalizante em funcionamento nesse conjunto de revoluções discutidas"15 (SKOCPOL, 1979, p. 6, tradução nossa). A partir do trecho citado, é possível depreender pelo menos três pontos relevantes à presente discussão. Primeiramente, ao sugerir que o seu uso de estudos de caso não tem finalidade exclusivamente narrativa, ela sugere que eles não são do tipo descritivo. Em segundo lugar, a autora destaca o propósito generalizante de sua análise, sugerindo que os estudos de caso em sua pesquisa têm por objetivo propor uma hipótese sobre as causas das revoluções. Finalmente, e derivado do ponto anterior, Skocpol deixa claro que cada um de seus casos devem ser entendidos como uma instância de um fenômeno mais abrangente: as revoluções em geral. Dito de outro modo, ao enunciar seus objetivos de pesquisa, poder-se-ia dizer que a autora estaria respondendo, em seus próprios termos, à pergunta "este caso é um caso de quê?".

Resumidamente, em se entendendo o tipo de objetivo da pesquisa, reconhecendo que não há finalidade descritiva e, por fim, uma vez que as hipóteses mais abrangentes que se busca gerar ou testar estejam claras para o próprio pesquisador, é possível passar a uma outra reflexão, que envolve entender quais seriam as possíveis causas do fenômeno que se deseja estudar.

13 Além disso, seria possível dizer que para os estudos de caso descritivos não existiria necessariamente um problema ou dificuldade em se selecionar casos, pois eles já teriam sido selecionados a priori, de acordo com as preferências do pesquisador (SEAWRIGHT e GERRING, 2008, p. 296).

14 Coincidentemente ou não, esses também seriam os dois tipos de estudo de caso que mais poderiam contribuir para o desenvolvimento teórico em uma determinada disciplina (BENNETT, 2004, p. 21).

15 No original, "not merely with narrating the cases one by one but primarily with understanding and explaining the generalizable logic at work in the entire set of revolutions under discussion". 
Elemento 4: Refletir sobre as (possíveis) causas do fenômeno que se deseja estudar

A decisão de tratar exclusivamente dos processos de seleção relacionados a estudos de caso que visam gerar e que buscam testar hipóteses (excluindo-se, portanto, os estudos de casos descritivos) também permite que se note um outro fator em comum nessas duas derivações do método: ambas se referem a estudos de caso causais. Esse enfoque aproxima a presente discussão de autores como Seawright e Gerring (2008, p. 296), por exemplo, que restringem suas técnicas de seleção de casos a estudos que apresentam algum tipo de inferência causal; assim como de autores que se colocam no campo mais pluralista das interpretações sobre metodologia, como Beach e Pedersen (2016, p. 4). E é amparado nesses últimos que uma conceitualização mais precisa de "caso" pode ser apresentada para referenciar o presente tópico: "uma instância da ocorrência de um processo causal, ligando uma causa (ou um conjunto de causas) a um resultado"16 (BEACH; PEDERSEN, 2016, p. 5, tradução nossa).

Considerando essa lógica causal, dois elementos interrelacionados passam a ser fundamentais antes que se possa selecionar um ou mais casos: definir quais condições fazem com que um caso possa ser considerado como uma instância apta a ser estudada e, em seguida, verificar quais dos possíveis casos atendem a essas condições. Este tópico refere-se ao primeiro desses passos, isto é, trata do processo no qual o pesquisador poderia obter ganhos de rigor ao refletir sobre quais causas possivelmente poderiam gerar determinados resultados, já que seria impraticável selecionar casos sem que pelo menos um dos lados desse sistema causal estivesse bem definido. Nessa mesma linha, Maoz (2002, p. 171) argumenta que uma das principais dificuldades de se construir um desenho de pesquisa que envolva estudos de caso é justamente a necessidade de empreender um processo de raciocínio retroativo que tente estabelecer uma explicação anterior para um dado resultado. Ou ainda, em termos mais simples e metafóricos, isso significa que "se nós não sabemos se estamos lidando com um jogo de baseball ou de futebol, nós não podemos selecionar um conjunto relevante de jogos (casos) para estudar"17 (BEACH; PEDERSEN, 2016, p. 248-249).

16 No original, "an instance of a causal process playing out, linking a cause (or set of causes) with an outcome".

17 No original, "if we do not know whether we are dealing with a game of baseball or football, we cannot select a relevant set of games (cases) to study". 
Nesse sentido, cabe ao pesquisador propor algumas possíveis causas que possam explicar o fenômeno mais geral de pesquisa que se deseja entender e, para alcançar tal finalidade, a principal recomendação é a de que seja feita uma busca na literatura já existente pelas possíveis relações causais sobre o tema de pesquisa (BEACH; PEDERSEN, 2016, p. 249), e por teorias preestabelecidas que possam ajudar na compreensão do fenômeno em questão (LEUFFEN, 2007, p. 151).

Para além dessa busca inicial, contudo, é importante que a sistematização das informações obtidas por meio da referência a outras obras não acabe por criar uma estrutura de causalidade predefinida. Isto é, deve existir uma margem de possibilidade para que o próprio pesquisador possa especular sobre supostos mecanismos causais que possam estar em operação, algo que pode ser feito mediante a realização de um processo de brainstorming (BEACH; PEDERSEN, 2016, p. 111), por exemplo. A principal recomendação para esse processo de elucubração é relativamente simples: deve prevalecer o julgamento do pesquisador sobre o que pode ser potencialmente relevante (KING; KEOHANE; VERBA, 1994, p. 52).

Por fim, vale ressaltar que a compreensão das causas e resultado do fenômeno durante a reflexão deste elemento constituinte da seleção de casos pode vir a ser meramente transitória e, por essa razão, é importante reforçar a noção de que as causas propostas neste momento são meramente possíveis. Isso porque esse processo de consolidação de uma população de casos envolve uma iteração entre o conhecimento preexistente apontado pela literatura e os dados coletados pelo pesquisador empiricamente, algo que acaba lembrando o conhecido dilema do ovo ou da galinha: "alguma teoria é sempre necessária antes da coleta de dados e alguns dados são necessários antes de qualquer teorização"18 (KING; KEOHANE; VERBA, 1994, p. 46, tradução nossa). Não é incomum, nesse sentido, que o pesquisador tenha que repensar suas propostas de causalidade e voltar à presente etapa assim que as primeiras compreensões do fenômeno começarem a ser obtidas.

Isso dito, e definidas as possíveis causas para o fenômeno que se deseja estudar com estudos de caso, a próxima seção visa tratar da identificação

18 No original, "some theory is always necessary before data collection and some data are required before any theorizing". 
de quais casos possivelmente se enquadram nessas condições, de modo a poder constituir uma população de casos a partir da qual a seleção de casos será realizada.

\section{Elemento 5: Consolidar uma população de casos}

Consolidar uma população de possíveis casos é fundamental para poder selecioná-los. Como afirmam Seawright e Gerring (2008, p. 296), se muito pouco ou praticamente nada se sabe sobre os casos, quaisquer técnicas de seleção encontrarão dificuldades de aplicação prática. Este componente do processo de seleção de casos, portanto, complementa o elemento anterior ao sugerir a avaliação e a definição de quais casos poderiam ser enquadrados nas causas e nos resultados relacionados à pesquisa proposta, o que, por sua vez, resultará em uma população de casos aptos a serem selecionados para um estudo mais aprofundado.

Antes, porém, de se iniciar a coleta de dados para as variáveis que são consideradas relevantes para a pesquisa, é de suma importância que sejam delimitados os principais recortes de dados, como o espacial e o temporal (BEACH; PEDERSEN, 2016, p. 136). Seja por razões inerentes ao interesse do pesquisador, como a busca por compreensão de um fenômeno em uma região em particular do mundo ou em um período específico da história; seja por razões meramente pragmáticas, como escassez de recursos, tempo ou financiamento ${ }^{19}$; é fundamental que a delimitação do escopo e abrangência do conjunto de dados esteja claro tanto para o pesquisador quanto para os futuros leitores (KING; KEOHANE; VERBA, 1994, p. 51), considerando a significativa possibilidade de um conjunto de dados incompleto enviesar os resultados de uma pesquisa (HUG, 2003).

Na sequência, é preciso compreender que o objetivo primordial pretendido como resultado deste componente da seleção de casos é um conjunto de dados, que deve, de maneira resumida, ser composto por três partes constituintes: os casos (ou unidades de análise), as condições causais supostas (ou variáveis) e as observações (o valor medido ou a adequação aos conceitos causais propostos) (KING; KEOHANE; VERBA, 1994, p. 51). Esquematicamente,

19 As quais, vale dizer, não são consideras justificativas metodologicamente válidas. Ainda assim, este ponto é digno de menção, considerando que não é incomum que pesquisadores justifiquem a delimitação de sua população de casos utilizando-se desses critérios (SEAWRIGHT; GERRING, 2008, p. 295). 
isso poderia ser representado por uma simples tabela bidimensional, na qual os casos poderiam ser listados nas linhas; as condições causais poderiam ser dispostas nas colunas; e o cruzamento entre linhas e colunas representariam os valores de uma variável ou a adequação conceitual daquele caso a um conceito específico ${ }^{20}$.

O primeiro eixo, a unidade de análise (ou caso), em teoria já teria sido delimitado no momento em que a pergunta "este caso é um caso de quê?" fosse respondida. Ainda assim, trata-se de uma ocasião importante para verificar se o fenômeno que se pretende estudar está alinhado conceitualmente com o fenômeno que se busca ter uma compreensão mais ampla, já que esse descasamento é relativamente comum nesta etapa da pesquisa. Mencionando um exemplo, Yin (2001, p. 44-45) sugere que é recorrente, por parte de pesquisadores, confundir estudos de caso de bairros com estudos de caso de pequenos grupos, e essa pequena diferença na definição da unidade de análise pode impactar significativamente na estratégia de coleta de dados - pois existiriam diferentes pesquisas e fontes de dados sobre bairros e sobre pequenos grupos, por exemplo.

Quanto ao segundo eixo - as possíveis causas do fenômeno de interesse do pesquisador -, esse também poderia ser considerado um ponto supostamente pacificado por reflexões anteriormente abordadas, mas algumas considerações adicionais necessitam ser feitas sobre a terminologia da causalidade empregada por diferentes autores. É comum que alguns autores, como King, Keohane e Verba (1994, p. 77), por exemplo, relacionem as causas de um fenômeno a "variáveis independentes" e os resultados a "variáveis dependentes", enquanto outros, como Beach e Pedersen (2016, p. 98-99), refiram-se a "condições causais" ao tratar das causas de um fenômeno, assim como prefiram empregar somente o termo "resultados" em detrimento de "variáveis dependentes". Essa diferenciação, que agruparia alguns autores em um grupo da "abordagem orientada por dados" e outros em um segundo grupo da "abordagem orientada por conceitos"21, não é meramente terminológica, pois ela impactaria na forma como as observações obtidas a partir dos dados seriam mensuradas e, consequentemente, no terceiro dos elementos da elaboração de uma população de casos.

20 Para alguns exemplos ilustrativos, ver Beach e Pedersen (2016, p. 236-244).

21 No original, "data-driven approach" e "concept-driven approach", respectivamente (BEACH; PEDERSEN, 2016, p. 102 , tradução nossa). 
Ainda que não seja objetivo do presente artigo posicionar-se favorável ou contrariamente a uma ou outra dessas duas abordagens, é fundamental que os pesquisadores, no decorrer da construção de sua população de casos, saibam em qual desses grupos sua lógica causal estaria amparada. Para os adeptos de uma abordagem orientada por dados, por exemplo, um dos principais desafios relacionados à mensuração de uma variável estaria na escolha da escala numérica (como nominal, ordinal, intervalos etc.) e dos valores numéricos que corresponderiam às supostas causas do fenômeno; enquanto que para aqueles que optam por uma abordagem orientada por conceitos o principal desafio seria definir os limiares qualitativos que demarcariam se um caso poderia ser considerado enquadrado ou não dentro de um determinado conceito causal (BEACH; PEDERSEN, 2016, p. 125-126).

Feitas essas considerações, a construção da população de casos envolve, fundamentalmente, a obtenção de informações sobre as mesmas possíveis causas do fenômeno que se deseja estudar (sejam elas dados numéricos ou considerações binárias do tipo "sim" ou "não" para adequação a conceitos causais) para todos os casos mapeados dentro dos recortes preestabelecidos. Quanto aos métodos envolvidos na coleta de dados para essa finalidade, eles poderiam ser os mais variados possíveis, tais quais entrevistas, surveys, experimentos, etnografia, dados históricos de fontes secundárias, análise de conteúdo, dentre outros (KING; KEOHANE; VERBA, 1994, p. 51).

Por fim, duas observações com relação a este emento do processo de seleção de casos devem ser feitas. A primeira delas é que o enquadramento de casos a causas nem sempre é intuitivo, tanto para variáveis numéricas quanto para conceitos causais. Sendo assim, por maiores que sejam os esforços empreendidos pelos pesquisadores, sempre existirá algum grau de incerteza residual sobre a adequação de um caso a uma explicação causal, especialmente na delimitação do limiar de adesão dos valores numéricos ou conceitos causais ao caso. Isso significa que na medida em que não seja possível justificar e documentar, com relativa confiança, que um caso deva pertencer a uma dada população, ele não deve fazer parte dela (BEACH; PEDERSEN, 2016, p. 131-133). Em segundo lugar, e assim como mencionado no componente sobre as possíveis causas do fenômeno de interesse do pesquisador, o processo iterativo entre teoria e dados também impacta a constituição da população, de tal maneira 
que os limites da população de casos podem ser expandidos ou retraídos com o decorrer do desenvolvimento da pesquisa (BEACH; PEDERSEN, 2016, p. 245).

Dito isso, e considerando que o pesquisador logrou estabelecer uma população de casos organizada com as informações relativas às possíveis causas do fenômeno que se deseja estudar, é possível, por fim, proceder a um dos últimos elementos relacionados ao processo de seleção de casos, isto é, a escolha de casos para estudo aprofundado.

\section{Elemento 6: Escolher casos propositalmente}

Escolher casos para representar instâncias de um fenômeno mais abrangente pode ser mais complexo do que inicialmente se supõe, e isso se deve a dois principais fatores: um relacionado ao pragmatismo da escolha de casos; e o segundo referente à possibilidade de uso da aleatoriedade na seleção de casos.

O primeiro desses fatores envolve o acúmulo e o desenvolvimento metodológico no que diz respeito a estudos de caso, que teria delimitado uma mudança na maneira como esse processo vinha sendo feito anteriormente. Como argumenta Levy (2008, p. 7), justificativas de caráter pragmático que outrora eram aceitas como válidas para a seleção de casos - como "interesse intrínseco" ou "relevância histórica" - passaram a ser preteridas, abrindo espaço para critérios mais rigorosos e transparentes sugeridos pela literatura metodológica.

$\mathrm{Na}$ esteira dessas novas recomendações, alguns autores passaram a sugerir que a maneira mais adequada para se escolher casos seria com a utilização de técnicas preexistentes relacionadas à estatística, como a seleção aleatória (SEKHON, 2004, p. 285). Essa sugestão estaria fundamentada na noção de que um dos principais problemas de estudos de casos e estudos comparados estaria no processo de escolha de casos, uma vez que os casos escolhidos teriam o potencial de afetar significativamente os resultados de uma pesquisa (GEDDES, 1990, p. 131). Em tese, tal problema, conhecido na literatura como viés de seleção, poderia ser facilmente solucionado ao se utilizar a seleção aleatória quando a população de casos disponível fosse relativamente grande, pois a aleatoriedade seria responsável por eliminar automaticamente esse viés de seleção (KING; KEOHANE; VERBA, 1994, p. 124).

Essa discussão, por sua vez, conduz a uma segunda dificuldade relativa ao processo de escolha de casos: nem sempre recorrer à aleatoriedade pode ser 
uma solução pertinente. Uma das principais reflexões nesse sentido provém de King, Keohane e Verba (1994, p. 125-126), ao reconhecerem que a seleção de caso aleatória poderia ser consideravelmente negativa tanto nos estudos que possuem uma população pequena de casos disponíveis (dada a possibilidade de casos relevantes para a argumentação serem deixados de fora da amostra) quanto nas pesquisas de natureza qualitativa (dada a comum indefinição dos limites de uma população de casos nessa circunstância). Contudo, e para além desse argumento, Seawright e Gerring (2008, p. 295-296) defendem que a seleção aleatória na realidade tenderia a ser prejudicial também em outras situações, isto é, não somente nas circunstâncias descritas por King, Keohane e Verba (1994), mas também em estudos de caso com finalidade causal que envolvam uma grande população de casos e cuja natureza seja eminentemente quantitativa.

Considerando esses dois desafios, ou seja, a recomendação de não se selecionar casos a partir de critérios pragmáticos e os possíveis efeitos deletérios da seleção aleatória, a solução apontada pela literatura tem sido na direção da seleção proposital de casos (KING; KEOHANE; VERBA, 1994; LEUFFEN, 2007; LEVY, 2008; SEAWRIGHT; GERRING, 2008). Escolher propositalmente, no entanto, não significa que qualquer caso possa ser priorizado arbitrariamente, ou que arguições genéricas possam ser apresentadas para justificar as escolhas feitas (LEUFFEN, 2007, p. 145), mas sim que cada um dos casos escolhidos para representar instâncias de proposições mais abrangentes estejam meticulosamente alinhados aos objetivos propostos e às teorias mobilizadas pelos pesquisadores.

Tendo em vista que abordar individualmente as diferentes técnicas de escolha de cada um dos autores que discutem esse tema seria impraticável, em se considerando os objetivos do presente artigo ${ }^{22}$, é fundamental que se depreenda desta subseção pelo menos dois pontos. O primeiro envolve frisar, por razões já expostas, que a escolha proposital de casos metodologicamente embasada é preferível à aleatória e à pragmática. Já o segundo ponto trata de reconhecer que as principais dificuldades que eventualmente venham a ser sentidas durante a escolha de casos possivelmente estariam relacionadas com a desconsideração de algum dos elementos do processo de seleção

22 Para discussões sobre diferentes técnicas de escolha de casos, ver, por exemplo, Levy (2008) e Seawright e Gerring (2008). 
de casos (GEORGE; BENNETT, 2005, p. 83), o que pode exigir que o pesquisador considere realizar novas reflexões ou readequações em questões como o objetivo da pesquisa, a generalização proposta, as possíveis causas do fenômeno, ou a população de casos.

Uma vez que tenha sido lograda a realização da escolha dos casos, o processo mais amplo da seleção de casos em tese estaria finalizado. No entanto, um último elemento ainda precisa ser sopesado, pois sem ele grande parte dos esforços despendidos em prol de um maior rigor e precisão na seleção de casos podem ser em vão: a transparência com relação a como cada um dos elementos do processo de seleção de casos foi endereçado, algo que idealmente deveria ocorrer de maneira concomitante a cada uma das reflexões suscitadas.

Elemento 7: Comprometer-se com a transparência em todos os elementos da seleção de casos

Ainda que todo esse processo descrito anteriormente - que se estende desde as primeiras definições sobre a pergunta de pesquisa até a escolha dos casos - seja de fato posto à prática, um questionamento relevante ainda poderia ser feito: o que, além da própria consciência dos autores, impediria que eles escolhessem propositalmente as evidências que embasassem as descrições, interpretações ou inferências causais que defendem (MORAVCSIK, 2014, p. 50)?

Esse tema, que tem ganhado maior profusão em termos de produção acadêmica nos anos recentes, vem sendo acompanhado por um emergente consenso em torno de uma principal resposta: uma maior transparência na pesquisa poderia ajudar a evidenciar os possíveis vieses conscientes ou inconscientes dos pesquisadores (MORAVCSIK, 2014, p. 48). Com maior transparência, todos os pesquisadores, independentemente de sua tradição metodológica, poderiam contribuir para aumentar a credibilidade e a legitimidade de suas pesquisas e, consequentemente, de suas respectivas disciplinas como um todo (LUPIA; ELMAN, 2014, p. 20). Sendo assim, essa recomendação também seria aplicável para os estudos de caso e, inclusive, essa seria uma efetiva maneira de imunizar pesquisadores que empregam esse método contra possíveis e comuns críticas, que geralmente sugerem que os resultados obtidos com estudos de caso seriam enviesados (BEACH; PEDERSEN, 2016, p. 124). 
A transparência com relação ao processo de seleção de casos, nesse sentido, seria um sétimo e importante elemento do processo de seleção de casos, e sua presença em cada um dos elementos anteriormente mencionados possui um grande potencial de incremento no rigor das pesquisas nas ciências sociais que decidam pela utilização de estudos de caso. Por essa razão, torna-se válido retomar brevemente alguns dos elementos anteriormente citados para que seja possível analisar a contribuição da transparência em cada um deles.

Quanto aos três primeiros componentes da seleção de casos mencionados - isto é, aqueles relacionados à pergunta de pesquisa, tipo de objetivo e definição de uma instância de um fenômeno mais abrangente - uma mera explicitação dos objetivos do pesquisador poderia ser suficiente para promover a transparência. Considerando que o interesse de pesquisa de um cientista social seria majoritariamente determinado por suas inclinações pessoais e valores, ou seja, teriam uma origem pessoal e idiossincrática (KING; KEOHANE; VERBA, 1994, p. 14), poderia ser demasiado intrusivo exigir que um pesquisador explicitasse as razões que teriam o levado a optar por uma determinada temática. Contudo, como critério de demonstração de relevância, uma maneira de ser claro ao se refletir sobre esses elementos seria apresentando as principais contribuições sociais (sua importância no mundo real) e teóricas (sua importância para a literatura na qual a pesquisa se insere) (KING; KEOHANE; VERBA, 1994, p. 15) - algo que não se relaciona exclusivamente com o emprego de estudos de caso, mas que poderia contribuir significativamente com as investigações científicas de um modo geral.

Os demais elementos, por sua vez, merecem ser analisados individualmente, já que o compromisso com a transparência no processo de reflexão de cada um deles poderia gerar expressivos ganhos em se tratando da elaboração de novos exemplos de bons estudos de caso. Em consonância com esse detalhamento individual, um dos elementos da seleção de casos digno de menção é aquele que trata do processo de elucubração sobre as possíveis causas de um dado fenômeno. Um dos pontos que poderia ser mais bem esclarecido ao se refletir sobre esta questão seria a explicitação, por parte dos pesquisadores, do processo pelo qual as causas propostas de suas inferências foram determinadas. Nesse sentido, seria importante evidenciar, por exemplo, como as tipologias foram extraídas da literatura preexistente sobre o objeto de pesquisa, assim como 
apontar porque se embasou em uma ou outra teoria, inclusive evidenciando as explicações concorrentes para o mesmo fenômeno (LEUFFEN, 2007, p. 151).

O elemento que trata da consolidação da população de casos também poderia ter incrementos em termos de rigor ao se apresentar de maneira clara todas as tarefas desempenhadas que culminaram na população de casos, o que se iniciaria com um detalhamento da origem dos dados: de acordo com King, Keohane e Verba (1994, p. 51), a regra mais importante para qualquer tipo de coleta de dados é uma explicação sobre como os dados foram criados ou obtidos, e o processo como isso se deu. Em seguida, esforços poderiam ser empregados para evidenciar os limiares utilizados para detalhar a adequação de uma causa (seja ela representada na forma de um valor em uma variável ou a adequação a um conceito) a um caso. Isso porque, ainda que um pesquisador seja altamente diligente (e independentemente de sua tradição metodológica ser majoritariamente quantitativa ou qualitativa), sempre haverá um elevado grau de subjetividade nesse processo de enquadramento de causas a casos, de tal maneira que uma boa forma de garantir a confiabilidade de sua inferência causal seria permitindo que outros pesquisadores julgassem como esse processo foi realizado (BEACH; PEDERSEN, 2016, p. 121-123).

Como exemplo ilustrativo, convém retomar a anteriormente mencionada análise de Skocpol (1979) sobre as causas das revoluções e, mais especificamente, as críticas dirigidas a ela. Uma das mais conhecidas análises nesse sentido é a realizada por Geddes (1990, p. 144-145), sugerindo que, caso Skocpol tivesse inserido outros casos em sua população - nomeadamente alguns países latino-americanos -, suas conclusões sobre as causas das revoluções poderiam ter sido significativamente diferentes. Por sua vez, Sekhon (2004, p. 286287) analisa a crítica de Geddes a Skocpol e verifica que também essa argumentação teria sido feita sobre bases frágeis, considerando que Geddes também não apresenta claramente quais países latino-americanos teriam sido utilizados para amparar sua argumentação, nem como teria chegado naquela população de casos sugerida. Esse simples exemplo ilustra como a transparência dos procedimentos de consolidação da população - não necessariamente em prol da replicação, mas meramente para aumentar a compreensão e persuasão de uma pesquisa -, poderiam contribuir significativamente para o acúmulo de conhecimento (LUPIA; ELMAN, 2014, p. 22): se Skocpol (1979) e Geddes 
(1990) tivessem descrito explicitamente seus processos de consolidação de casos, as discussões sobre a validade de suas argumentações provavelmente estariam se dando em outras bases.

Como complemento das discussões sobre a transparência nos processos de suposição de causas e consolidação da população, vale retomar também a possível mutabilidade presente ao se analisar esses elementos, que faz com que alguns autores entendam que a utilização de estudos de caso é, em grande medida, um processo de constante reconstrução (MAOZ, 2002, p. 171). Na medida em que se consolida uma população de casos, mais se sabe sobre as particularidades dos casos estudados e sobre a relevância (ou irrelevância) de algumas causas incialmente supostas, o que pode vir a exigir uma revisão em algumas dessas causas. Ao se alterar as possíveis causas, no entanto, a população de casos também é alterada, o que gera um processo retroativo de alterações que somente se encerra quando existe uma ideia clara por parte do pesquisador de que as observações mensuradas nos dados refletem o que se pretende inferir (BEACH; PEDERSEN, 2016, p. 120). Quanto a esse ponto, seria fundamental que os pesquisadores que optassem pela utilização estudos de caso passassem a ser especialmente transparentes quanto a essas "idas e vindas" entre hipóteses e dados, já que se corre o risco de que ambos os componentes do processo de seleção de casos sejam tidos como enviesados caso não exista uma grande clareza tanto sobre o que foi incialmente concebido quanto ao que foi posteriormente modificado no decorrer da pesquisa.

Finalmente, um dos últimos elementos, que envolve a escolha de fato dos casos que serão analisados a partir da população, também é passível de obter ganhos em termos de rigor na medida em que explicações pormenorizadas de como esse processo foi realizado fossem dadas. Ainda que sejam válidas as críticas que sugerem que a seleção de casos poderia induzir os resultados pretendidos ao se selecionar a partir das variáveis dependentes (GEDDES, 1990, p. 132) - ou, em outras palavras, selecionar especificamente os casos que melhor exemplificam o que se deseja argumentar com uma pesquisa -, também é fundamental que se reconheça que é praticamente impossível selecionar casos sem que exista algum tipo de viés, consciente ou inconsciente (KING; KEOHANE; VERBA, 1994, p. 141). Mais uma vez, portanto, a sugestão sobre o que pode ser feito aponta na direção a uma 
maior transparência: "nossos mecanismos de seleção de casos devem ser evidenciados da maneira mais transparente possível, para que o leitor possa estimar a magnitude e a as direções do possível viés"23 (LEUFFEN, 2007, p. 158, tradução nossa).

Com essa última recomendação, fica evidente que a transparência é um elemento adicional e fundamental a ser incorporado em cada um dos componentes da seleção de casos, desde as primeiras definições sobre o uso ou não de estudos de caso (de acordo com a pergunta de pesquisa) até a efetiva escolha dos casos (de acordo com as técnicas utilizadas). E, uma vez delimitados esses sete elementos relacionados a uma rigorosa seleção de casos, é possível enfim proceder às considerações finais.

\section{Considerações finais}

O presente artigo teve como principal objetivo apresentar aos cientistas sociais que considerem adequado utilizar estudos de caso em suas pesquisas sete elementos que poderiam ser mobilizados para selecionar casos de maneira rigorosa: avaliação da adequação da utilização de estudos de caso à pergunta principal de pesquisa; avaliação do tipo de objetivo de pesquisa; reflexão sobre a pergunta "este caso é um caso de quê"; análise sobre as causas do fenômeno de estudo; consolidação de uma população de casos; escolha proposital de casos; e o comprometimento do pesquisador de garantir a transparência durante o processo de seleção de casos.

Sendo os estudos de caso um método extremamente valioso de exploração e compreensão de fenômenos sociais, é inegável que a subutilização, utilização inadequada ou sem o rigor necessário comprometem sua credibilidade enquanto instrumento de geração de pesquisas cientificamente críveis. Por essa razão, a sistematização da literatura preexistente aqui realizada visou não somente fomentar a reflexão sobre um método específico, de modo a colaborar com o desenvolvimento de novos estudos de caso que possam servir de referência em termos de confiabilidade nas ciências sociais brasileiras, mas também pretendeu instigar um debate mais amplo sobre como a busca por rigor e transparência nas ciências sociais poderia envolver um número mais amplo de pesquisadores, discorrendo sobre este e outros métodos.

23 No original, "our case selection mechanisms should be made as transparent as possible so that the reader can estimate the magnitude and directions of possible bias". 
Para além dessas contribuições, é fundamental reconhecer que o artigo também pode possuir limitações. Dentre estas, talvez a mais significativa seja o possível viés de confirmação relacionado à escolha dos autores utilizados para empreender a revisão bibliográfica, já que foram selecionados majoritariamente acadêmicos que igualmente defendem a aptidão da seleção proposital de casos. Ainda assim, espera-se que a principal estratégia concorrente para se selecionar casos, isto é, aquela baseada na aleatoriedade, tenha tido suas principais fragilidades expostas durante a explanação sobre a escolha de casos, o que sustentaria a predileção pelos autores aqui referenciados.

Ainda assim, mesmo em um cenário no qual eventuais críticas a este artigo sejam consideradas procedentes, tais contestações podem contribuir significativamente para o desenvolvimento da ciência brasileira. Isso porque, para superar o "calcanhar metodológico" das ciências sociais do Brasil, não basta sugerir que um ou outro método deva prevalecer, ou que um ou outro método deva ser ensinado: é necessário que exista uma intensa discussão sobre como melhorar a aplicação e a utilização de cada uma das diversas escolhas metodológicas que um pesquisador pode ter à sua disposição. Que este artigo, portanto, seja apenas uma das inúmeras e bem-vindas contribuições a esse crescente debate em prol de um maior rigor nas pesquisas sociais do país.

\section{Referências}

ALVES-MAZZOTTI, Alda Judith. Usos e abusos dos estudos de caso. Cadernos de Pesquisa, São Paulo, v. 36, n. 129, p. 637-651, 2006.

BARBERIA, Lorena Guadalupe; GODOY, Samuel Ralize de; BARBOZA, Danilo Praxedes. Novas perspectivas sobre o 'calcanhar metodológico': o ensino de métodos de pesquisa em ciência política no Brasil. Teoria \& Sociedade, Belo Horizonte, v. 2, n. 22, p. 156-184, 2014.

BAUMEISTER, Roy F.; LEARY, Mark R. Writing narrative literature reviews.

Review of General Psychology, Washington, D.C., v. 1, n. 3, p. 311-320, 1997. BEACH, Derek; PEDERSEN, Rasmus Brun. Causal case study methods: foundations and guidelines for comparing, matching and tracing. [S. l.]: University of Michigan Press, 2016.

BENNETT, Andrew. Case study methods: design, use, and comparative advantages. In: SPRINZ, Detlef F.; WOLINSKY-NAHMIAS, Yael. Models, 
numbers and cases: methods for studying international relations. Ann Arbor: University of Michigan Press, 2004. p. 19-55.

BORGES, André. Desenvolvendo argumentos teóricos a partir de estudos de caso: o debate recente em torno da pesquisa histórico-comparativa. BIB Revista Brasileira de Informações Bibliográficas em Ciências Sociais, São Paulo, n. 63, p. 45-59, 2007.

FLYVBJERG, Bent. Five misunderstandings about case-study research. Qualitative Inquiry, Thousand Oaks, v. 12, n. 2, p. 219-245, 2006.

GEDDES, Barbara. Selection bias in comparative politics: how the cases you choose affect the answers you get. Political Analysis, Oxford, v. 2, p. 131150, 1990.

GEORGE, Alexander L.; BENNETT, Andrew. Case studies and theory development in the Social Sciences. Cambridge: MIT Press, 2005.

GSCHWEND, Thomas; SCHIMMELFENNIG, Frank. Introduction: designing research in political science: a dialogue between theory and data. In: GSCHWEND, Thomas; SCHIMMELFENNIG, Frank (ed.). Research design in political science: how to practice what they preach. New York: Palgrave Macmillan, 2007. cap. 1, p. 3-18.

HUG, Simon. Selection bias in comparative research: the case of incomplete data sets. Political Analysis, Oxford, v. 11, n. 3, p. 25-274, 2003.

KING, Gary; KEOHANE, Robert O.; VERBA, Sidney. Designing social inquiry: scientific inference in qualitiative research. Princeton: Princeton University Press, 1994.

LEUFFEN, Dirk. Case selection and selection bias in small-n research. In: GSCHWEND, Thomas; SCHIMMELFENNIG, Frank (ed.). Research design in political science: how to practice what they preach. New York: Palgrave Macmillan, 2007. cap. 3, p. 145-160.

LEVY, Jack S. Case studies: types, designs, and logics of inference. Conflict Management and Peace Science, Philadelphia, v. 25, n. 1, p. 1-18, 2008.

LUPIA, Arthur; ELMAN, Colin. Openness in political science: data access and research transparency. PS - Political Science \& Politics, Washington, D.C., v. 47, n. 1, p. 19-42, 2014.

MAOZ, Zeev. Case study methodology in international studies: from storytelling to hypothesis testing. In: HARVEY, Frank P.; BRECHER, Michael 
(ed.). Evaluating methodology in international studies. [S. l.]: University of Michigan Press, 2002. p. 161-186.

MORAVCSIK, Andrew. Transparency: the revolution in qualitative research. PS

- Political Science \& Politics, Washington, D.C., v. 47, n. 1, p. 48-53, 2014. MORSE, Janice. Reframing rigor in qualitative inquiry. In: DENZIN, Norman K.;

LINCOLN, Yvonna S. (ed.). The SAGE Handbook of Qualitative Research.

5. ed. Thousand Oaks: SAGE Publications, 2018. cap. 35, p. 1373-1409.

NEIVA, Pedro. Revisitando o calcanhar de Aquiles metodológico das ciências sociais no Brasil. Sociologia, Problemas e Práticas, Lisboa, n. 79, p. 65-83, 2015.

RAGIN, Charles C. Introduction: cases of "what is a case?". In: RAGIN, Charles C.; BECKER, Howard S. (ed.). What is a case?: exploring the foundations of social inquiry. Cambridge: Cambridge University Press, 1992. p. 1-17. SCHWANDT, Thomas A.; GATES, Emily F. Case study methodology. In: DENZIN, Norman K.; LINCOLN, Yvonna S. (ed.). The SAGE Handbook of Qualitative Research. 5. ed. Thousand Oaks: SAGE Publications, 2018. Cap. 14, p. 600-630.

SEAWRIGHT, Jason; GERRING, John. Case selection techniques in case study research: a menu of qualitative and quantitative options. Political Research Quarterly, Salt Lake City, v. 62, n. 2, p. 294-308, 2008.

SEKHON, Jasjeet S. Quality meets quantity: case studies, conditional probability, and counterfactuals. Perspectives on Politics, Cambridge, v. 2, n. 2, p. 281-293, 2004.

SKOCPOL, Theda. States and social revolutions: a comparative analysis of

France, Russia and China. Cambridge: Cambridge University Press, 1979. SOARES, Gláucio A. D. O calcanhar metodológico da ciência política no Brasil.

Sociologia, Problemas e Práticas, Lisboa, n. 48, p. 27-52, 2005.

TRACY, Sarah J. Qualitative quality: eight “big-tent” criteria for excellent qualitative research. Qualitative Inquiry, Thousand Oaks, v. 16, n. 10, p. 837-851, 2010. VITORINO, R.; OLIVEIRA, L. Estudos de caso na ciência política: uma análise dos artigos publicados nas Revistas Dados e RBCS. In: ENCONTRO NACIONAL DA ASSOCIAÇÃO BRASILEIRA DE CIÊNCIA POLÍTICA, 8., 10-4 ago. 2012, Gramado. Anais [...]. Gramado: FURGS, 2012. p. 1-16. Disponível em: https://cienciapolitica.org.br/web/system/files/documentos/ 
eventos/2017/02/estudos-caso-ciencia-politica-analise-dos-artigos-publicados.pdf. Acesso em: 30 jul. 2021.

YIN, Robert K. Estudo de caso: planejamento e métodos. Tradução de Daniel Grassi. 2. ed. Porto Alegre: Bookman, 2001.

\section{Sete elementos do processo de seleção de casos: contribuições para um maior rigor e transparência nas Ciências Sociais}

Resumo: Este artigo, direcionado aos pesquisadores que considerem adequada a utilização de estudos de caso em suas pesquisas, visa apresentar, de maneira sistematizada, os sete principais elementos relacionados ao procedimento de seleção de casos - isto é, o processo que se inicia com a pergunta de pesquisa e que se encerra com a escolha dos casos propriamente dita, sendo perpassada por uma necessidade latente de garantia de transparência. Para tal finalidade, é empreendida uma revisão bibliográfica narrativa com a finalidade de organizar, de maneira intuitiva, alguns elementos que se encontram dispersos pela literatura que trata do assunto. Com isso, busca-se contribuir tanto com o fortalecimento do uso rigoroso dos estudos de caso quanto com o desenvolvimento de um debate mais amplo sobre a necessidade de maior transparência e sistematização no que tange ao emprego de diferentes métodos nas ciências sociais brasileiras.

Palavras-chave: estudos de caso; metodologia; seleção de casos; rigor científico; transparência.

\section{Seven elements of the case selection process: contributions towards more rigor and transparency in the Social Sciences}

Abstract: This article, directed at researchers who consider it appropriate to use case studies in their investigations, aims to present, in a systematic way, the seven key elements related to the case selection procedure - that is, the process that begins with the research question and that ends with the case selection itself, while dealing with the latent need to guarantee transparency along the way. To this end, a narrative literature review is conducted in order to organize and to present in an intuitive manner elements scattered throughout the literature on case studies. The purpose is to contribute to a more rigorous use of case studies and to the development of a broader debate on the need 
for more transparency and systematization in the use of different methods in Brazilian Social Sciences.

Keywords: case studies; methods; case selection; scientific rigor; transparency.

\section{Siete elementos del proceso de selección de casos: contribucio-} nes a un mayor rigor y transparencia en las Ciencias Sociales

Resumen: Este artículo, dirigido a investigadores que consideren oportuno utilizar estudios de casos en sus investigaciones, tiene como objetivo presentar de manera sistemática los siete principales elementos relacionados con el procedimiento de selección de casos - o, en otras palabras, el proceso que comienza con la pregunta de investigación y que finaliza con la elección de los casos propiamente dichos, atravesado por una necesidad latente de garantizar la transparencia. Para ese propósito, se realiza una revisión bibliográfica narrativa con el objetivo de organizar de una manera intuitiva algunos elementos que se encuentran dispersos en la literatura de lo asunto. Con ello, lo que se busca es contribuir tanto con el fortalecimiento del uso riguroso de los estudios de caso como con el desarrollo de un debate más amplio sobre la necesidad de que exista una mayor transparencia y sistematización en el uso de diferentes métodos en las ciencias sociales brasileñas.

Palabras clave: estudios de caso; metodología; selección de casos; rigor científico; transparencia.

Recebido em 01 de setembro de 2020

Aprovado em 09 de abril de 2021. 